\title{
Syndrome de Gorlin-Goltz : à propos d'un cas
}

\section{Gorlin-Goltz syndrome: report of a case}

\author{
MOHAMED RACHID GHAILAN, HANANE BENHALIMA, GHAFOUR RABEH, ABDELILAH OUJILAL, \\ ANAS BENBOUZID, RAZIKA BENCHEIKH, LEILA ESSAKALI, MOHAMED BOULAICH, MOHAMED KZADRI
}

\section{RÉSUMÉ}

Le syndrome de Gorlin et Goltz est une affection héréditaire, à transmission autosomique dominante, due à une mutation du gène PTCH ayant pour localisation chromosomique 9q 22.3-q31. C'est un syndrome rare qui se caractérise par un spectre d'anomalies du développement et par une prédisposition à différents cancers. La complexité des signes cliniques a conduit à établir des critères spécifiques pour faciliter le diagnostic. La prise en charge thérapeutique est symptomatique, avec une surveillance carcinologique rigoureuse. Nous rapportons une nouvelle observation de syndrome de Gorlin et Goltz chez un adolescent de 17 ans et, à travers une revue de littérature, nous discutons les principales caractéristiques de cette rare affection. Med Buccale Chir Buccale 2007; 13: 97-101.

mots clés: syndrome de Gorlin-Goltz, kératokyste odontogénique, nævomatose basocellulaire

médecine buccale chirurgie buccale

VOL. $13, N^{\circ} 2$ 2007

page 97

\section{SUMMARY}

Gorlin syndrome, also known as nevoid basal cell carcinoma syndrome (NBCCS), is a hereditary condition transmitted as an autosmal dominant trait with high penetrance and variable expressitivity, caused by mutation in the PTCH gene mapped to chromosome 9q 22.3-q31. It is characterized mainly by basal cell carcinomas, odontogenic keratocysts and skeletat anomalies. More than 100 minor criteria have been described, but 2 major and 1 minor criteria or 1 major and 3 minor criteria are necessary for the diagnosis. A case of Gorlin-Goltz syndrome has been reported with good illustration and review of literature. Med Buccale Chir Buccale 2007; 13: 97-101.

key words: Gorlin-Goltz syndrome, odontogenic keratocyst, nevoid basal cell carcinoma 
médecine buccale chirurgie buccale

VOL. $13, \mathrm{~N}^{\circ} 2$ 2007

page 98
Le syndrome de Gorlin et Goltz, appelé encore naevomatose basocellulaire (NBC) est une affection héréditaire rare, à transmission autosomique dominante, à pénétrance complète et à expressivité variable. Elle se caractérise par un spectre d'anomalies de développement et une prédisposition à différents cancers. Ce syndrome est du à des mutations sur un gène suppresseur de tumeur ayant comme localisation chromosomique 9q 22.3-q31 [1,2].

Ce terrain justifie un dépistage précoce et une surveillance prolongée des patients et de leur descendance.

\section{OBSERVATION}

A A, adolescent de 17 ans, sans antécédents pathologiques particuliers, a été opéré en mars 2004 par son dentiste pour deux petits kystes situés entre les racines de 33 et 34 , et de 41 et 42 , puis il nous a été adressé pour prise en charge de deux volumineux kystes maxillaires.

Lors de l'examen maxillo-facial, on découvre un faciès longiligne avec une saillie des bosses frontales, un hypertélorisme, un prognathisme modéré et de multiples petits naevi disséminés sur le visage, également présents sur le reste du corps. Les oreilles sont normalement ourlées et implantées (Fig. 1). L'examen endobucal montre deux volumineux kystes maxillaires droit et gauche, bombant dans le vestibule, responsables de malpositions dentaires intéressant 16, 17, 23, et 24. La muqueuse buccale est d'aspect normal. Le reste de l'examen somatique, neurologique, ophtalmologique et orthopédique ne décèle aucune anomalie, la puberté s'est effectuée normalement. II n'y a pas de déficit mental. Le bilan radiologique (orthopantomogramme, incidence de Blondeau, examen tomodensitométrique du massif facial) a mis en évidence du coté gauche, un très volumineux kyste maxillaire uniloculaire à limites nettes ; les dents adjacentes sont refoulées en périphérie. II existe également du coté droit, un volumineux kyste comblant entièrement le sinus maxillaire droit (Fig. 2). L'ensemble de ces signes (kystes multiples des maxillaires, naevi basocellulaires, anomalies faciales) constitue le syndrome des kystes des maxillaires.
Figure 1 : Dysmorphie faciale et nombreux naevi disséminés sur le visage et le reste du corps

Facial dysmorphia associated to facial and thoracic multiple naevi

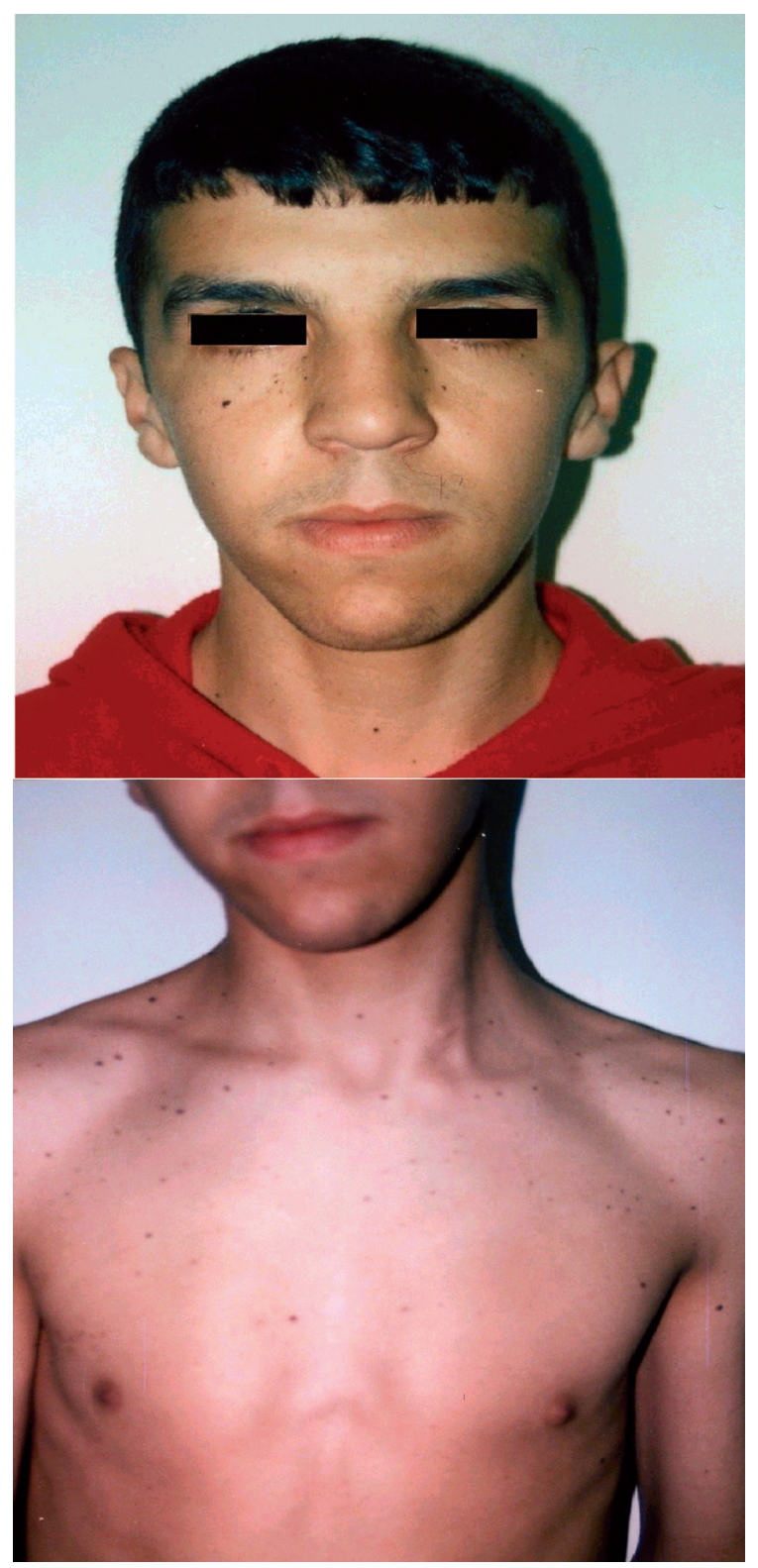

Un bilan spécifique a été réalisé pour compléter le dossier : la radiographie du poignet a montré un âge osseux voisin de 17 ans et une minéralisation osseuse normale, les radiographies du squelette n'ont pas objectivé de malformations squelettiques, l'examen tomodensitométrique cérébral a mis en évidence une calcification de la faux de cerveau (Fig. 3). L'enquête génétique n'a pas trouvé de cas similaires dans la famille. 
Figure 2 : Image scannographique montrant deux kystes maxillaires avec déplacement de dents adjacentes CT scan showing maxillary cysts with teeth displacement

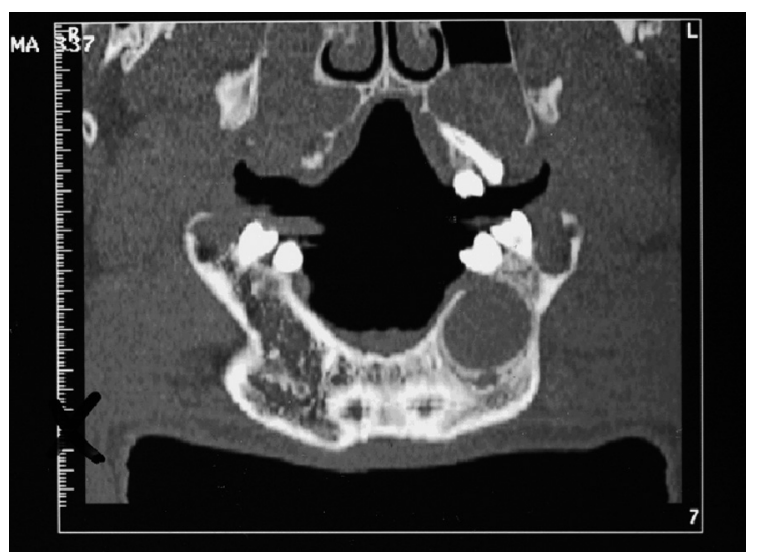

Figure 3 : Examen tomodensitométrique cérébral montrant des calcifications intracrâniennes (faux de cerveau)

Brain CT scan showing intracranial calcifications (cerebral falx)

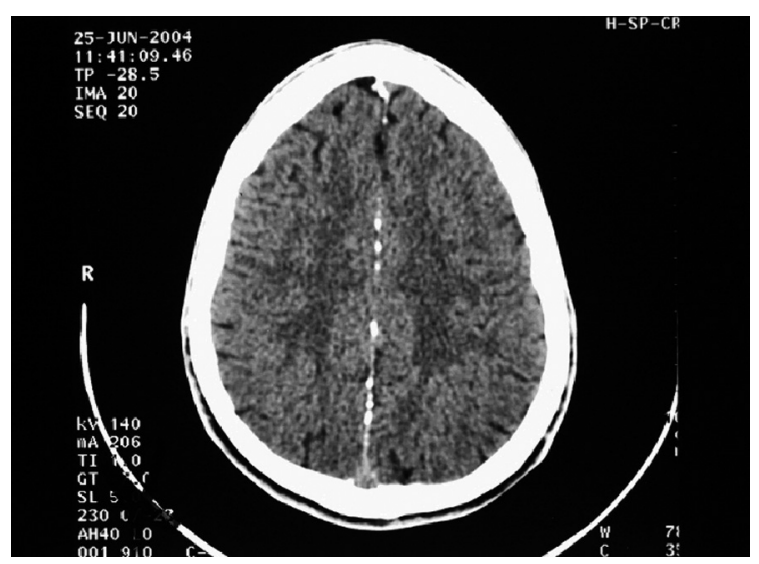

L'intervention faite le 25/04/2004 a consisté en une énucléation totale des deux kystes maxillaires ; cette intervention n'a présenté aucune difficulté. L'examen anatomopathologique des pièces opératoires a confirmé le diagnostic de kératokyste, donc celui de syndrome de Gorlin et Goltz. Un orthopantomogramme de contrôle, réalisé 6 mois plus tard, a montré l'absence de récidives et la bonne reconstruction osseuse.

\section{DISCUSSION}

Le syndrome de Gorlin et Goltz est une affection génétique autosomique dominante [1,2]. La première description a été faite en 1894 par Jarisch mais c'est Gorlin et Goltz qui, en 1960, définissent l'affection qui portent leur nom. Et depuis, plus de 700 cas ont été rapportés dans la littérature $[1,2,3]$.

La prévalence de ce syndrome a été diversement estimée, elle est d'environ 1/60000 dans la population générale ; il est retrouvé chez $0,5 \%$ des patients atteints de carcinomes baso-celullaires [4]. Le risque d'avoir un enfant atteint est de $50 \%$ dans la descendance [4]. Les hommes et les femmes sont atteints dans les mêmes proportions. La transmission est autosomique dominante, avec une pénétrance de $97 \%$ et une expressivité variable ${ }^{[1,2,4]}$. Cependant, des cas sporadiques ont été rapportés. Entre 5 et $50 \%$ des patients présentent des néomutations et, dans plus de $60 \%$ des cas, aucun autre membre de la famille n'est atteint $[4,5,8]$.

Le gène responsable du syndrome a été localisé sur le chromosome 9 en 9q22.3-q31 en 1996 [4,5,9]. II s'agit du gène Patched (PTCH), supposé avoir un rôle anti-oncogène. Selon Knudson en 1971, il y a une perte des deux allèles de ce gène (perte de l'hétérozygotie) avec une première mutation germinale, responsable du syndrome malformatif, qui prédispose à la survenue de tumeurs, et la deuxième mutation (somatique) qui entraîne le développement de la tumeur. Le gène PTCH est l'homologue d'un gène de segmentation de la drosophile intervenant dans la transduction du signal médié par Hedgehog. Cette voie est impliquée à de multiples reprises au cours de développement où PTCH code pour une glycoprotéine transmembranaire qui pourrait fonctionner comme récepteur ou transporteur. La majorité des mutations identifiées dans le gène PTCH chez les patients sont des mutations non-sens qui aboutissent à la synthèse d'une protéine défective tronquée.

Cliniquement, cette affection est caractérisée par un spectre d'anomalies de développement et une prédisposition à différents cancers ${ }^{[1,2,4]}$. Elle se traduit par de nombreux naevi baso-cellulaires, des médecine buccale chirurgie buccale VOL. $13, \mathrm{~N}^{\circ} 2$ 2007 page 99 
médecine buccale chirurgie buccale

VOL. $13, \mathrm{~N}^{\circ} 2$ 2007

page 100 kératokystes odontogéniques des maxillaires, une hyperkératose palmo-plantaire, des anomalies de squelette, des calcifications ectopiques intracrâniennes et une dysmorphie faciale évocatrice ; un retard mental est observé chez $5 \%$ des patients $[2,3,7]$. Ces manifestations cliniques sont age-dépendants ${ }^{[2,3,4]}$. Certains signes sont présents dès la naissance, d'autres apparaissent progressivement au cours de la deuxième décennie.

Sur le revêtement cutané, les naevi baso-cellulaires sont retrouvés sur tout le corps, sauf les extrémités inférieures. Ce sont des papules, de la même couleur que la peau ou brun pâle, de 1 à $10 \mathrm{~mm}$ de diamètre. Ils se transforment en carcinomes baso-cellulaires habituellement entre l'âge de la puberté et 35 ans ${ }^{[4,9]}$. Les kératokystes odontogéniques sont présents chez $75 \%$ des patients, parfois comme seule manifestation au cours de la première décennie. Ils sont souvent bilatéraux ou multiples, volumineux et récidivants $[4,9,12]$. La complexité des signes cliniques retrouvés dans ce syndrome a conduit à établir des critères spécifiques pour faciliter le diagnostic. II implique de retrouver chez un patient au moins deux des quatre critères majeurs ou un critère majeur et deux critères mineurs $[2,4,8]$.

Critères majeurs :

- kératokystes odontogéniques

- porokératose (pitts) palmo-plantaire

- carcinomes basocellulaires

- calcifications intracrâniennes (faux de cerveau)

Critères mineurs :

- macrocéphalie, bosses frontales, hypertélorisme

- prognathisme, palais ogival, grains de milium

- kystes épidermiques

- anomalies osseuses: costo-vertébrales, thoraciques

- raccourcissement des $4^{\text {es }}$ métacarpiens

- fibromes ovariens, médulloblastomes.
Le diagnostic différentiel se pose peu en dehors de quelques syndromes dermatologiques rares, comme le syndrome de Bazex qui associe des carcinomes basocellulaires à une hypotrichose, une hypohidrose et une atrophodermie folliculaire.

Sur le plan thérapeutique, il n'existe pas actuellement de traitement spécifique pour cette affection. Leur prise en charge est symptomatique avec une surveillance carcinologique particulière, impliquant l'intervention de différents spécialistes. La protection solaire est impérative et les patients doivent être contrôlés régulièrement, en particulier un examen dermatologique doit être effectué tous les deux et trois mois. L'enquête génétique est indispensable pour la détection de nouveau cas dans la famille. Un examen neurologique s'impose annuellement chez les enfants jusqu'à l'âge de sept ans afin de déceler un médulloblastome.

\section{CONCLUSION}

Le syndrome de Gorlin et Goltz est une affection génétique, rare, multisystémique, à transmission autosomique dominante. Il est défini classiquement par la triade composée de naevi baso-cellulaires, de kératokystes des maxillaires et de malformations squelettiques. Ce syndrome est du à des mutations sur un gène suppresseur de tumeur ayant 9q22.3-q31 comme localisation chromosomique.

Le potentiel carcinologique de ce syndrome fait sa gravité, justifiant un dépistage précoce et une surveillance régulière et prolongée des patients et de leur descendance. Le traitement reste simplement symptomatique. 


\section{RÉFÉRENCES}

1 - Compton JG, Goldstein AM. Fine mapping of the locus for nevoid basal cell carcinoma syndrome on chromosome 9q. J Invest Dermatol 1994 ; 103 : 178-81.

2 - Bale AE, Gailani MR, Leffel DJ. Nevoid basal cell carcinoma syndrome. J Invest Dermatol 1994 ; 103(suppl) : 126S-130S.

3 - Lambrescht JT, Kreusch T. Examine your orofacial cleft patients for Gorlin-Goltz syndrome. Cleft Palate Craniofac J 1996 ; 34 : 342-50.

4 - Lacombe D. Le syndrome de Gorlin. Encyclopédie Orphonet, février 1997.

5 - Daley TD, Wysocki GP, Pringle GA. Relative incidence of odontogenic tumors and oral jaw cysts in a canadian population. Oral Surg Oral Med Oral Patol 1994 ; 77 : 276-80.
6 - El-Hajj G, Anneroth G. Odontogenic keratocysts-a retrospective clinical and histologic study. Int J Oral Maxillofac Surg 1996 ; 25 : 124-9.

7 - Rivet JV, Servant M, Monteil JP. Syndrome de GorlinGoltz à propos d'un kyste maxillaire. Rev Stomatol Chir Maxillofac 2000 ; 101 : 194-6.

8 - Gorlin RJ, Goltz RW. Multiple nevoid basal cell epithelioma, jaw cysts and bifid rib syndrome. N Engl J Med $1960 ; 262$ : 908-12.

9 - Panté P, Beauvais P, Marie MP. Un nouveau cas de naevomatose basocellulaire (syndrome de Gorlin-Goltz). Rev Stomatol Chir Maxillofac 1978 ; 79 : 411-8.

10 - Amlashi SFA, Riffaud L, Brassier G, Morandi X. Nevoid basal cell carcinoma syndrome: relation with desmoplastic medulloblastoma in infancy. A population-based study and review of the literature. Cancer 2003 ; 98 : 618-24. médecine
buccale
chirurgie
buccale

VOL. $13, \mathrm{~N}^{\circ} 2$ 2007

page 101 